\title{
A Novel Sparse Polynomial Expansion Method for Interval and Random Response Analysis of Uncertain Vibro-Acoustic System
}

\author{
Shengwen Yin $(\mathbb{D}$, Xiaohan Zhu $(\mathbb{D}$, and Xiang Liu $(\mathbb{B})$ \\ Key Laboratory of Traffic Safety on Track, Ministry of Education, School of Traffic \& Transportation Engineering, \\ Central South University, Changsha, China \\ Correspondence should be addressed to Xiang Liu; xiangliu@csu.edu.cn
}

Received 13 June 2021; Revised 27 August 2021; Accepted 31 August 2021; Published 24 September 2021

Academic Editor: Antonio Batista

Copyright $(92021$ Shengwen Yin et al. This is an open access article distributed under the Creative Commons Attribution License, which permits unrestricted use, distribution, and reproduction in any medium, provided the original work is properly cited.

For the vibro-acoustic system with interval and random uncertainties, polynomial chaos expansions have received broad and persistent attention. Nevertheless, the cost of the computation process increases sharply with the increasing number of uncertain parameters. This study presents a novel interval and random polynomial expansion method, called Sparse Grids' Sequential Sampling-based Interval and Random Arbitrary Polynomial Chaos (SGS-IRAPC) method, to obtain the response of a vibroacoustic system with interval and random uncertainties. The proposed SGS-IRAPC retains the accuracy and the simplicity of the traditional arbitrary polynomial chaos method, while avoiding its inefficiency. In the SGS-IRAPC, the response is approximated by the moment-based arbitrary polynomial chaos expansion and the expansion coefficient is determined by the least squares approximation method. A new sparse sampling scheme combined the sparse grids' scheme with the sequential sampling scheme which is employed to generate the sampling points used to calculate the expansion coefficient to decrease the computational cost. The efficiency of the proposed surrogate method is demonstrated using a typical mathematical problem and an engineering application.

\section{Introduction}

The vibro-acoustic analysis is fundamental for the vibration and noise control of a vibro-acoustic system in vehicles such as cars, high-speed trains, and airplanes in the early stages of the design [1]. The traditional numerical methods used for the vibro-acoustic analysis are generally based on the assumptions of deterministic parameters. Nevertheless, the uncertainties related to the vibro-acoustic system, such as the material properties, the boundary conditions, and the aggressive environment factors, are inevitable in the practical engineering applications due to the inevitable manufacturing errors and incomplete knowledge. The response of the vibroacoustic system is highly sensitive to the uncertain parameters, and the results obtained from the traditional numerical methods based on deterministic hypothesis may be unreliable without considering these uncertainties. As a result, there is an increasing demand for the development of an uncertainty analysis method in the vibro-acoustic analysis.
Random uncertainty and epistemic uncertainty are two typical types commonly employed in uncertainty analysis. The probabilistic methods are widely used for problems with random uncertainty and generally consist of Monte Carlo (MC) method [2-4], stochastic perturbation approach [5-8], and polynomial chaos expansion (PCE) approach $[9,10]$, and so on. The uncertain parameters of the probabilistic method are always defined by random variables with sufficient probabilistic characteristics. The epistemic uncertainty is another type of uncertainty with lacking probabilistic information. The probabilistic methods are not suitable for the epistemic uncertainty model when the probabilistic characteristic of the uncertain parameters is insufficient to get. Nonprobabilistic methods have been became an efficient alternative to effectively model the epistemic uncertainty. The typical nonprobabilistic method includes the interval analysis [11-13], the fuzzy theory $[14,15]$, the evidence theory [16-18], and the $p$-box set $[19,20]$. Among these methods, the interval method has 
gained widespread attention because of its conceptual simplicity [21]. Various methods have been established in the previous studies conducted on the uncertainty quantification of the interval model, such as the interval perturbation approach $[22,23]$, the interval Chebyshev approach [24-27], the interval collocation approach [28], and the optimization-based approach [29].

As explained earlier, the probabilistic method or the interval method is employed to solve problems with random uncertainty or epistemic uncertainty, respectively. Nevertheless, in the complex vibro-acoustic system, random and epistemic uncertainty exist simultaneously [30-33]. The interval and random mixed model [34] has been proposed for problems related to both random and epistemic uncertainty. The hybrid interval and random analysis is much more complex and time consuming when compared to the pure interval or random analysis. The perturbation method is commonly used for the interval and random mixed model $[28,29]$. The hybrid perturbation method can reach great precision for the uncertain analysis of interval and random mixed uncertain model. However, it is only restricted to the uncertain problem with a small uncertainty level [24]. The PCE method exhibits more excellent accuracy for the hybrid uncertain problem with a large uncertainty level, when compared to the perturbation method. Yin et al. [35] employed the Gegenbauer polynomial in the framework of the Gegenbauer polynomial chaos to propose a unified PCE method and applied it to the uncertain analysis of the vibroacoustic system. However, it is only suitable for uncertain problems which the probability density functions (PDFs) of uncertain parameters are exact and continuous. Yin et al. [36] proposed a unified arbitrary polynomial expansion method. The computational accuracy of hybrid uncertain analysis for the vibro-acoustic problem with arbitrary PDFs has been improved effectively. The arbitrary polynomial chaos expansion method presents better accuracy than the Gegenbauer polynomial chaos expansion method for the uncertain problem with arbitrary PDFs. This method is feasible depending on the availability of the PDFs of the random variables. However, in some cases, the given statistical data lacks probabilistic information [9], resulting in errors in the estimation of the probability distributions. Chen et al. [37] proposed a unified Interval and Random Moment-based Arbitrary Polynomial Chaos (IRMAPC) method to resolve this issue. This method constructs the polynomial basis corresponding to the random variable according to the moment of the random variable. It effectively avoids the generation of errors in the PDF assumption process. The Moment-based Arbitrary Polynomial Chaos (MAPC) presents better accuracy than the arbitrary polynomial chaos for the interval and random mixed uncertain model when only the statistical data of the random variable is available.

Despite the effectiveness of the MAPC expansion method in the interval and random uncertain analysis, an important problem still remains unresolved. For multivariable problems, the sampling points, which are used to calculate the expansion coefficient of this method, are the tensor product of the Gaussian integration points. The multidimensional uncertainties are usually included in the complex vibro-acoustic system [38]. This makes the computing cost of the MAPC method tremendous when obtaining the response of the vibro-acoustic system. The traditional arbitrary polynomial expansion method involves a substantial computing cost when is used to analyze the complex vibro-acoustic problems. Therefore, it is important to decrease the computing cost involved in the calculation of the expansion coefficient.

To improve the computing efficiency, various sampling methods with fewer sampling times have been developed to determine the coefficient of the PCE method. Traditional sampling methods can be considered as "one-shot" sampling methods, such as the Latin Hypercube Sampling (LHS) [39] and Orthogonal Sampling [40] and the Central Composite Design [41]. Since the sampling points in the "one-shot" sampling method are chosen only once, the "one-shot" sampling method is inflexible and incomplete during the analysis of the response surface. A sequential sampling method $[42,43]$ has been recently proposed to improve the flexibility and to provide complete sampling. The sequential sampling method improves on the "one-shot" sampling methods. In this method, the required sampling points are selected from the candidate points, which are the tensor product of the Gaussian integration points for a multivariable problem. In the sequential sampling method, the number of integration points in the candidate set increases sharply as the number of variables increases. This phenomenon leads to excessive computational costs.

Overall, the arbitrary Polynomial Chaos expansion method is unsuitable for the vibro-acoustic system involving multidimensional problems of interval and random uncertainties, despite its significant contribution to the hybrid uncertainty analysis. The sequential sampling method [43] has been used to decrease the computing cost of the multivariable problem. However, the existing sequential sampling scheme has a poor sampling efficiency. Therefore, it is necessary to propose a new method which can reach the same level of precision as the MAPC method, while providing better computational efficiency.

This study focuses on the development of a new interval and random polynomial expansion method exhibiting high computational efficiency for a vibro-acoustic system with interval and random uncertainties, called the Sparse Grids' Sequential Sampling-based Interval and Random Arbitrary Polynomial Chaos (SGS-IRAPC). First, the unified IRMAPC method is introduced for the vibro-acoustic analysis. Second, the sparse grids' sequential sampling scheme, which combines the sequential sampling method with the sparse grids' method, is proposed to decrease the computing cost in the surrogate method construction. In this sampling method, the sampling points, which are used to calculate the coefficient of the PCE method, are selected form integration points in the candidate set. A sparse grids' method based on the Smolyak algorithm is adopted to select the integration points of the candidate set to greatly enhance the computing efficiency. The proposed method is compared with the IRMAPC and the Sequential Sampling Scheme-based Interval and Random Arbitrary Polynomial Chaos (SSS- 
IRAPC). The effective property of the proposed surrogate modeling method is demonstrated using two numerical examples.

\section{Basic Theory of Interval and Random Moment-Based Arbitrary Polynomial Chaos}

The Interval and Random Moment-based Arbitrary Polynomial Chaos (IRMAPC) method [37, 44, 45] is briefly summarized in this section.

According to the basic concept of the Moment-based Arbitrary Polynomial Chaos (MAPC) expansion method, a function, $F(x)$, can be approximated by the MAPC as

$$
F(x)=\sum_{i=0}^{N} f_{i} G_{i}(x),
$$

in which $N$ represents the retained order, the expansion coefficient, and the polynomial basis and $G_{i}(x)$ is obtained from the moment of the random variable. The detailed procedure to determine the polynomial basis, $G_{i}(x)$, is provided in [37, 45].

In [37], the MAPC method is applied to the interval and random analysis in a unified form. The random vector, $x^{R}$, and the interval vector, $x^{I}$, are defined as follows:

$$
\begin{aligned}
\mathbf{x}^{R} & =\left[x_{1}^{R}, x_{2}^{R}, \ldots, x_{L_{1}}^{R}\right], \\
\mathbf{x}^{I} & =\left[x_{1}^{I}, x_{2}^{I}, \ldots, x_{L_{2}}^{I}\right],
\end{aligned}
$$

where $x_{i}^{R}\left(i=1,2, \ldots, L_{1}\right)$ is the $i$ th random variable, $x_{i}^{I}(i=$ $1,2, \ldots, L_{2}$ ) denotes the $i$ th interval variable, and $L_{1}$ and $L_{2}$ are the number of random variables and interval variables, respectively. All the uncertain parameters are then denoted by a vector, $\mathbf{x}=\left[\mathbf{x}^{R}, \mathbf{x}^{I}\right]$. The total number of variables is denoted as $L, L=L_{1}+L_{2}$.

For multivariate problems, a straightforward approach to extend the interpolation function from one variable problem to multivariable problems is to use the tensor product method [46]. Based on the MAPC expansion, $F(x)$ of the interval and random variables can be approximated by the following formula:

$$
\begin{aligned}
F & =F\left(x^{I}, x^{R}\right) \\
& =\sum_{i_{1}=0}^{N_{1}} \cdots \sum_{i_{L}=0}^{N_{L}} f_{i_{1}, \ldots, i_{L}} G_{i_{1}, \ldots, i_{L_{1}}}\left(x^{I}\right) G_{i_{L_{1}+1}, \ldots, i_{L_{2}}}\left(\mathbf{x}^{R}\right),
\end{aligned}
$$

where

$$
\begin{aligned}
G_{i_{1}, \ldots, i_{L_{1}}}\left(\mathbf{x}^{R}\right) & =\prod_{k=1}^{L_{1}} G_{i_{k}}\left(x_{k}^{R}\right), \\
G_{i_{L_{1}+1}, \ldots, i_{L}}\left(\mathbf{x}^{I}\right) & =\prod_{k=L_{1}+1}^{L} G_{i_{k}}\left(x_{k}^{I}\right) .
\end{aligned}
$$

In the above equation, $G_{i_{k}}\left(x_{k}^{R}\right)$ and $G_{i_{k}}\left(x_{k}^{I}\right)$ denote the $i_{k}$ order polynomial basis related to the $k$ th random variable and the $k$ th interval variable, respectively. For the random variable, $G_{i_{k}}\left(x_{k}^{R}\right)$ is determined by the moment of the $k$ th random variable. For the interval variable, $G_{i_{k}}\left(x_{k}^{I}\right)$ is orthogonal to the weight function of the Chebyshev polynomial. From the application of the MAPC for random analysis, it is observed that when the moment is set as $\mu_{k}=\int_{-1}^{1} x^{k} \rho(x)$, the polynomial is orthogonal and is denoted by $\rho(x)$. It indicates that the polynomial basis is orthogonal to the weight function of the Chebyshev polynomial if the moment is determined by

$$
\begin{aligned}
\mu_{k} & =\int_{-1}^{1} x^{k} w_{C}(x) \mathrm{d} x \\
& =\int_{-1}^{1} x^{k} \frac{1}{\pi \sqrt{1-x^{2}}} \mathrm{~d} x,
\end{aligned}
$$

where $w_{C}(x)=1 /\left(\pi \sqrt{1-x^{2}}\right)$ is the weight function of the Chebyshev polynomial. After the moment is determined, the polynomial basis for interval analysis can be calculated by using equations (4) and (5).

The expansion coefficient, $f_{i_{1}, \ldots, i_{L}}$, is calculated by the Gauss integration rule [37, 45]. Normally, the number of the Gaussion integration points related to $i$ th variable is set as $M_{i}=N_{i}+1$. Furthermore, the sampling points, which are used to calculate the expansion coefficient of this method, are also the tensor product of the Gaussian integration points. For multivariable problems, the total number of the integration points required to determine $f_{i_{1}, \ldots, i_{L}}$ is shown as follows:

$$
N_{\text {tot }}=\left(N_{1}+1\right) \times\left(N_{2}+1\right) \times \cdots \times\left(N_{L}+1\right) .
$$

For multivariable problems, the IRMAPC expansion method as equation (3) is truncated by the tensor order expansion. And, the number of the expansion coefficient of the IRMAPC expansion method is as equation (6).

It is observed from the above equation that the total number of the integration points and the expansion coefficient $f_{i_{1}, \ldots, i_{L}}$ are exponentially increased with the increase in both retained order and number of variables. Therefore, the IRMAPC expansion method involves considerable computational cost for an uncertain problem when either the number of uncertain variables or the retained order of polynomial basis is relatively large. In order to solve this problem and improve the efficiency of the traditional arbitrary polynomial expansion method, a new surrogate method is proposed in the next section.

\section{SGS-IRAPC Method}

The sequential sampling scheme has been widely used to calculate the coefficient of a polynomial expansion [42, 43]. Generally, the candidates and the selection scheme of the sampling points greatly influence the accuracy and the efficiency of the sequential sampling scheme-based polynomial expansion method. This section presents the sparse grids' sequential sampling scheme to calculate the coefficient of the IRMAPC expansion. In the proposed sequential sampling scheme, the integration points of sparse grids' 
quadrature [46-48] are used as the candidates of the sampling method, while the sequential sampling scheme, used in [49], is used to select the sampling points from the candidates. A new method called the SGS-IRAPC method is proposed based on the developed sequential sampling scheme.

3.1. The Expansion of the SGS-IRAPC. In order to improve the precision of the IRMAPC expansion method, a simple format is adopted in the IRMAPC expansion method to reduce the number of the expansion coefficients $[49,50]$. Equation (3) can be rewritten as

$$
\begin{aligned}
F & =F\left(x^{I}, x^{R}\right) \\
& =\sum_{0 \leq i_{1}+\cdots+i_{L_{1}}+\cdots+i_{L} \leq n} f_{i_{1}, \ldots, i_{L}} G_{i_{1}, \ldots, i_{L_{1}}}\left(x^{I}\right) G_{i_{L_{1}+1}, \ldots, i_{L_{2}}}\left(x^{R}\right),
\end{aligned}
$$

where $L$ is the total number of variables, $L=L_{1}+L_{2}$. $L_{1}$ and $L_{2}$ are the number of random variables and interval variables, respectively. Therefore, the number of the expansion coefficients can be evinced as the following formula:

$$
N_{c}(n, L)=\frac{(n+L) !}{L ! n !} .
$$

Consequently, the number of the expansion coefficients to be calculated is reduced from $N=\left(N_{1}+1\right) \times\left(N_{2}+1\right) \times$ $\cdots \times\left(N_{L}+1\right)$ to a small amount, $N_{C}$, and the computational efficiency of the IRMAPC expansion method for the multidimensional uncertain problems is improved.

$f_{i_{1}, \ldots, i_{L}}$ of the IRMAPC expansion method can be calculated by using the least squares approach (LSA) [25]. Equation (3) is simplified as

$$
\begin{aligned}
F(x) & =\boldsymbol{\beta}^{T} \boldsymbol{\alpha}, \\
\boldsymbol{\beta} & =\left[\beta_{1} \ldots \beta_{s}\right]^{T} \\
& =\left[f_{0 \ldots 0}, \ldots, f_{i_{1} \ldots i_{L}}\right]^{T}, \quad 0 \leq i_{1}+\cdots+i_{L} \leq n, \\
\boldsymbol{\alpha} & =\left[\alpha_{1} \ldots \alpha_{s}\right]^{T} \\
& =\left[G_{0 \ldots 0}, \ldots, G_{i_{1} \ldots i_{L}}\right]^{T} .
\end{aligned}
$$

In the above equation, $\beta$ is a coefficient vector, $\alpha$ is a polynomial basis vector, and $s$ represents the number of the expansion coefficients of the IRMAPC expansion method and is equal to the number of the polynomial basis of the IRMAPC expansion method, $s=N_{c}$. The coefficient vector, $\beta$, which is calculated by the LSA, is expressed by the following formula:

$$
\begin{aligned}
\boldsymbol{\beta} & =\left(\mathbf{A}^{T} \mathbf{A}\right)^{-1} \mathbf{A}^{T} \mathbf{Y}, \\
\mathbf{Y} & =\left[y\left(\mathbf{x}_{1}^{I}, \mathbf{x}_{1}^{R}\right) \ldots y\left(\mathbf{x}_{s}^{I}, \mathbf{x}_{s}^{R}\right)\right], \quad s=N_{C}, \\
A & =\left[\begin{array}{ccc}
\alpha_{1}\left(x_{1}^{I}, x_{1}^{R}\right) & \ldots & \alpha_{s}\left(x_{1}^{I}, x_{1}^{R}\right) \\
\vdots & \ddots & \vdots \\
\alpha_{s}\left(x_{s}^{I}, x_{s}^{R}\right) & \ldots & \alpha_{s}\left(x_{s}^{I}, x_{s}^{R}\right)
\end{array}\right], \quad s=N_{C},
\end{aligned}
$$

where the element of the vector, $\mathbf{Y}$, constitutes the values of the function, $y(x)$, which are calculated at the sampling points; the matrix, A, consists of the polynomial basis of the IRMAPC expansion at the integration points; $\left(x_{1}^{I}, x_{1}^{R}\right), \ldots,\left(x_{s}^{I}, x_{s}^{R}\right)$ denotes the integration points. Moreover, the matrix, A, in (9) must be a full column rank matrix.

\subsection{Candidates of the Sparse Grids' Sequential Sampling} Scheme. The LHS is generally used to determine the candidates of the sampling method. However, the LHS-based method might not present a stable convergence as the candidates are generated randomly. Subsequently, the integration points of the Gaussian quadrature have been employed as the candidate points to improve the stabilization for the sampled method. The Gaussian quadraturebased sampling method can generally stabilize the convergence with the increase in the sampling points. However, the number of integration points of the Gaussian quadrature increases rapidly as the number of uncertain parameters increases. Essentially, there will be a large number of candidates for the numerical analysis when the number of uncertain parameter is relatively large. Consequently, the optimal selection of the sampling points from the candidates becomes tedious.

The sparse grids' quadrature is an alternative integration technique used to calculate the expansion coefficient. The integration points of the sparse grids' quadrature are used as the candidates of the proposed sparse sampling scheme to reduce the candidates of sampling. The sparse grids' quadrature uses a special linear combination of the tensor product operations of the one-dimensional Gaussian integration points to construct a multidimensional discrete sample space [51]. This section presents the procedure used to select the integration points by the sparse grids' scheme.

The arbitrarily continuous function can be approximated as $Q_{k}^{1}(f), k=0,1, \ldots, l$, by (1), in which $l$ is the retained order of the IRMAPC expansion method.

Based on the nested hierarchical principle of the Smolyak algorithm [51], the difference format of the approximated function is

$$
\Delta_{k}^{1}(f)=\left(Q_{k}^{1}-Q_{k-1}^{1}\right)(f), \quad Q_{0}^{1}(f)=0 .
$$

In addition, according to the Smolyak algorithm, the approximated function with the dimension $d$ and retained order $l$ can be contrasted as

$$
Q_{1}^{d}(f)=\sum_{|k| \leq l+d-1}\left(\Delta_{k_{1}}^{1} \otimes \cdots \otimes \Delta_{k_{d}}^{1}\right)(f),
$$

where $\otimes$ denotes the operation of the tensor product and $|k|$ denotes the sum of the multidimensional indicators $\left(|k|=\sum_{i=1}^{d} k_{i}, k_{i}=0,1, \ldots, l\right)$. Through the operation of the tensor product, (14) can be expressed as $[46,47,52,53]$ 


$$
Q_{1}^{d}(f)=\sum_{l+1 \leq|k| \leq l+d}(-1)^{l+d-|k|}\left(\begin{array}{c}
d-1 \\
l+d-|k|
\end{array}\right)\left(Q_{k_{1}}^{1} \otimes \cdots \otimes Q_{k_{d}}^{1}\right)(f) .
$$

Therefore, the integration points of the multidimensional problem in the sparse grids' sampling scheme can be defined as [47]

$$
U_{1}^{d}=\underset{l+1 \leq k \mid \leq l+d}{U}\left(U_{k_{1}}^{1} \otimes \cdots \otimes U_{k_{d}}^{1}\right) .
$$

From equation (16), we can state that the correlation between the dimension $d$ and the order $l$ is $l+1 \leq|k| \leq l+d$ $[46,47]$. The integration points in the sparse grids can be selected between the Gaussian points of order, $(l+1)$, and lower than order, $(l+d)$. The number of integration points in the sparse grids' sampling scheme is reasonably controlled within a specific range when compared to the integration points in the full factor grids. The sparse grids' sampling scheme can be used to avoid problems of the large number of integration points in the candidate set used to calculate the coefficient of the IRMAPC expansion method.

For example, considering the case $d=2$ and $l=2$, the process of construction is as Figure 1(a). According to the relationship of the inequality $l+1 \leq|k| \leq l+d$, all index combinations of $k_{i}$ can be expressed as $\left[k_{1}, k_{2}\right]=\{[1,2],[2,1],[1,3],[3,1],[2,2]\}$. To clearly illustrate the sparse grids' sampling scheme, the integration points in the sparse grids' sampling scheme are shown as the red points in Figure 1(b). Finally, the number of integration points in the sparse grids' sampling scheme is 13 , when the repetitive integration points have been removed.

The number of integration points in the sparse grids and the full factor mesh is listed in Table 1, from which it is observed that the advantages of the sparse grids' scheme become increasingly evident with the number of variables increases.

3.3. Sequential Sampling Scheme. In [49], the sequential sampling scheme-based on the maximin principle has been used in the multidimensional problem with interval and random uncertainties to improve the approximation efficiency.

3.3.1. The Homogenization of the Candidate Set. The integration points produced by the sparse grids' sampling scheme are used as the candidate set for the sampling sequential scheme. The candidate set generated according to (13)-(16) is shown in Figure 1. Essentially, the sequential sampling scheme is only suitable for the symmetric and uniformly distributed candidate set. However, as shown in Figure 1 , the candidate set in the $x$ space produced by the sparse grids' sampling scheme is not uniformly distributed. Therefore, a $\beta$ space is introduced, which is distributed uniformly, where both $\beta_{i}$ and $x_{i}$ denote the ith integration point in the candidate set. Figure 2 shows the distribution map of the $x$ space and the $\beta$ space for a case with $n=4$ and $L=2$.
3.3.2. The Initial Sampled Set. The initial sampled set greatly affects the sampling precision. Usually the points in the initial sampled set distributed uniformly are helpful to improve the sampling precision. According to [54], Xia and $\mathrm{Yu}$ adopted the maximin metric measure to assess the uniformity of the candidate set produced by the sparse grids' sampling scheme. The scalar-valued criterion function [55] shown in (17) is mainly used to rank the set of competing sampling:

$$
\Phi_{q}(\beta)=\left(\sum_{i=1}^{z_{0}} \sum_{j=i+1}^{z_{0}} d\left(\beta^{(i)}, \beta^{(j)}\right)^{-q}\right)^{1 / q},
$$

where $q$ is a relative large integer which is set as 100 in this study, $z_{0}$ denotes the number of integration points in the candidate set, and $\beta^{(i)}$ denotes the $i$ th integration point in the $\beta$ space; the Euclidean distance, $d\left(\beta^{(i)}, \beta^{(j)}\right)$, can be expressed as follows [49]:

$$
d\left(\beta^{(i)}, \beta^{(j)}\right)=\left(\sum_{k=1}^{L}\left|\beta_{k}^{(i)}-\beta_{k}^{(j)}\right|^{2}\right)^{1 / 2} .
$$

In equation (17), $k$ is the number of variables, $\beta_{k}^{(i)}(k=$ $\left.1,2, \ldots, L ; i=1,2, \ldots, m_{k}\right)$ is the $i$ th sampling points of the $k$ th variable; and 2 is the Euclidean norm in this study. The smaller the value of $\Phi_{q}$, the greater the uniformity of the sampled set. To ensure the uniformity of the initial sampling set, the sampling points should be located in all levels in each variable. The initial sampled set can be expressed as a matrix with a size of $L \times \mathrm{ml}$, where the row denotes the sequence of the initial sampling point and the column denotes the sequence of the variables. To simplify the notation, we use the levels (from 1 to $\mathrm{m}$ ) to denote the location of sampling points. The number of sampling points in the initial sampling set is $m l=m \times L$ where $m$ is the number of the integration points when the sparse sampling scheme is adopted for one-dimensional problems and is set as $m=1+2+\cdots+N$, where $N$ is the retained order of the polynomial. The first row is defined as the reference row. To ensure uniformity, the first $m$ elements of the first row are designed as $1,2, \ldots, m$. This definition ensures that all alternative integration points of the first variable have been included in the initial sampling set. The sampling point, whose sequence of the sampling points for the first variable is 1 , is defined as the first sampling point. For instance, in a case with $N=3, L=4$, and $m=10$, the matrix as the initial sampling set is given in Table 2. In this case, the first sampling point is set as $[1,4-5]$. The remaining elements in the initial sampled set are obtained by the minimum value of the maximin metric, $\Phi_{q}$. The sampled set is represented by $\gamma$, and the candidate set is represented by $\beta$. According to $[49,50], \Phi_{q}\left(\beta, \beta_{1}^{(j)}\right)$ can be simplified as follows: 


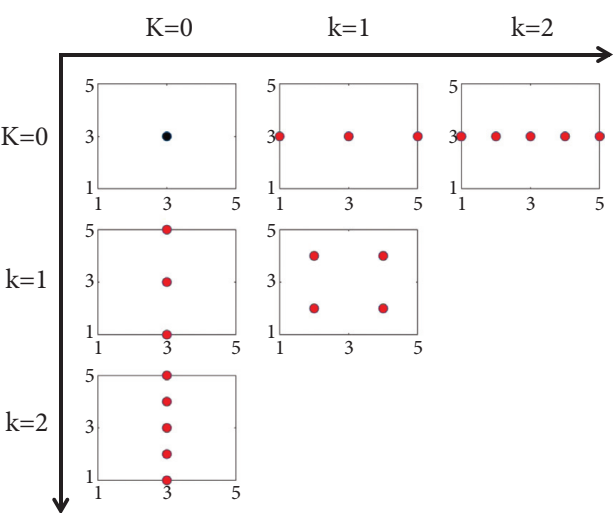

(a)

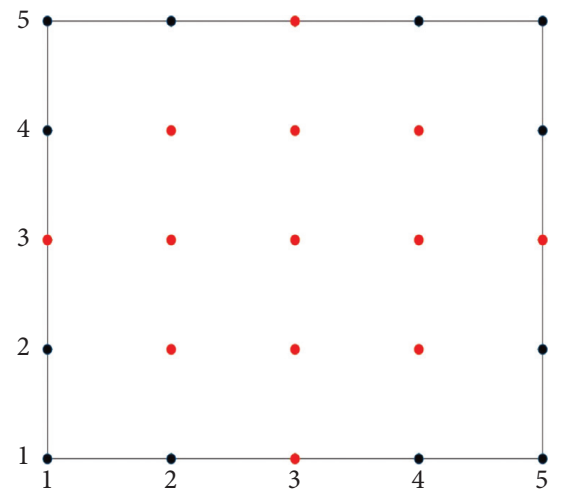

(b)

FIgURE 1: Integration points of sparse grids' sampling points at $d=2$ and $l=2$.

TABLe 1: Number of integration points in sparse grids or full factor mesh.

\begin{tabular}{lccc}
\hline No. of variables & Order of MAPC expansion & No. of integration points (sparse grids) & No. of integration points (full factor mesh) \\
\hline \multirow{4}{*}{5} & 1 & 11 & 32 \\
& 2 & 66 & 243 \\
& 3 & 286 & 1024 \\
& 4 & 1001 & 64 \\
6 & 1 & 13 & 729 \\
& 2 & 89 & 4096 \\
& 3 & 433 & 15625 \\
\hline
\end{tabular}

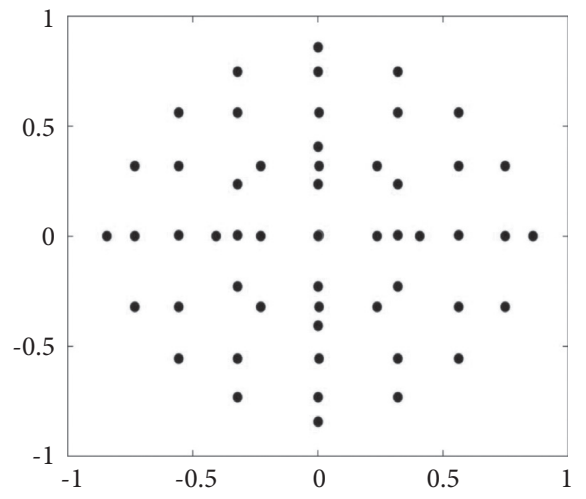

(a)

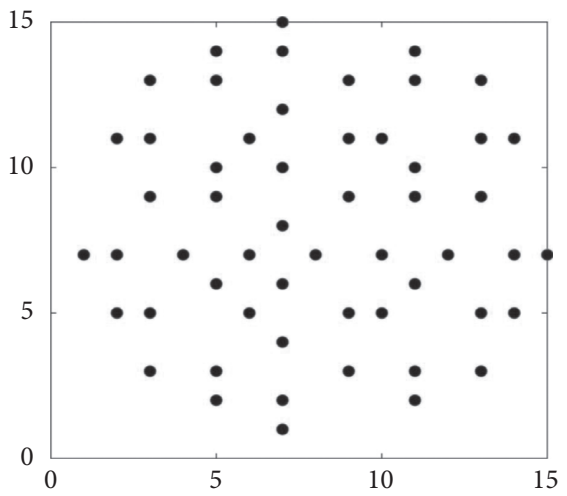

(b)

Figure 2: Candidate set in $x$ space and $\beta$ space for $n=4$ and $L=2$. (a) $x$ space; (b) $\beta$ space.

TABLe 2: The initial sampled set in $\beta$ space for $N=3, L=4$, and $m=10$.

\begin{tabular}{|c|c|c|c|c|c|c|c|c|c|c|}
\hline \multirow{2}{*}{ No. of variables } & \multicolumn{10}{|c|}{ No. of sampling points } \\
\hline & 1 & 2 & 3 & 4 & 5 & 6 & 7 & 8 & 9 & 10 \\
\hline 1 & 1 & 2 & 3 & 4 & 5 & 6 & 7 & 8 & 9 & 10 \\
\hline 2 & 5 & 5 & 7 & 5 & 3 & 5 & 5 & 7 & 5 & 5 \\
\hline 3 & 6 & 6 & 3 & 6 & 3 & 3 & 6 & 6 & 8 & 6 \\
\hline 4 & 5 & 8 & 5 & 5 & 8 & 5 & 5 & 8 & 5 & 5 \\
\hline
\end{tabular}

$$
\Phi_{q}\left(\beta, \beta_{1}^{(h)}\right)=\left(\sum_{i=1}^{s_{0}} d\left(\beta^{(i)}, \beta_{1}^{(h)}\right)^{-q}\right)^{1 / q}
$$

where $\left(\beta, \beta_{1}^{(h)}\right)$ represents new sampled set which includes the old sampling point $\beta^{(i)} \in \gamma$ and the new sampling point, $\beta_{1}^{(h)} \in \beta$ and $s_{0}$ represents the number of the sampling points in 
sampled set $\gamma$. The new sampling point with the minimum $\Phi_{q}$ is then included in the sampled set, $\gamma$. Each time a new sampling point is selected, the sampled set and candidate set must be updated to calculate $\Phi_{q}$ and to select the next sampling point. The process is repeated until the all elements from the second column to the $m$ th column are determined. To clearly demonstrate the sampling points in the initial sampled set, the results of the top $m$ column are presented in Table 2 .

To calculate the second sampling point, the second element in the second column should be determined according to (19) at first. In this case, when $\Phi_{q}$ gets the minimum value in a two-dimensional space constructed by the first and second variables, the level of the second value is 5 , which means the second element in the second column is 5. Similarly, the third element in the second column is obtained by minimizing in a three-dimensional space, which is 6 . When the second column is obtained, a new sampling point is selected from the candidate set to the initial sampling set. Then, update the initial sampling set, $\beta$, and the candidate set, $\gamma$. Repeat this operation until the other elements of the top column have been obtained.

To ensure the uniformity, the sampling points for each variable should distribute as uniformly as possible. To seek that the uniformity is guaranteed along each variable, the sampling points should be located in all alternative levels in each dimension. However, only the first row (the elements of the first variable) is uniform in Table 2. The first row is moved to the last, and the other rows are moved forward to solve this problem. The second row is then denoted as the reference row. The previous process is repeated to obtain the elements from the 11-th to the 20-th columns. $m$ new sampling points are obtained for each row moving operation. Furthermore, the operation of the row movement ensures that the initial sampling set obtains all alternative levels in every variable at least once. The row moving operation is carried out until the levels of each variable are distributed with sufficient uniformity in the initial sampled set after which the initial sampled set is obtained.

3.3.3. Avoiding Matrix Singularity. According to (10)-(12), $f_{i_{1}, \ldots, i_{L}}$ is calculated by using the LSA. The matrix, $\mathbf{A}$, of the polynomial basis used in the LSA must be a full column rank matrix. The singular value decomposition (SVD) is used to determine the rank of the matrix, A. The SVD of the matrix, $\mathbf{A} \in \mathbb{R}^{m \times n}$, is as follows [41]:

$$
\mathbf{A}=\mathbf{U S V}^{T} .
$$

In equation (20), $\mathbf{U} \in \mathbb{R}^{m \times m}$ and $\mathbf{V} \in \mathbb{R}^{n \times n}$ are orthogonal. The diagonal matrix, $\mathbf{S}$, is as follows:

$$
\mathbf{S}=\operatorname{diag}\left(\sigma_{1}, \ldots, \sigma_{L}\right) \in \mathbb{R}^{m \times n},
$$

where $L$ denotes the rank of the matrix, $\mathbf{A}, \sigma_{i}(i=1, \ldots, L)$ is the singular value of $\mathbf{A}$, and $\sigma_{1} \geq \sigma_{2} \geq \cdots \geq \sigma_{L} \geq 0$. The rank of matrix, $\mathbf{A}$, is equal to the number of singular values greater than a certain numerical tol cutoff. The tol can be determined as follows:

$$
\text { tol }=\max (\operatorname{size}(A)) \times|\max (S)| .
$$

When the rank of the matrix, $\mathbf{A}$, is equal to $n, \mathbf{A}$ is known as a full column rank matrix. Each new sampling point needs to calculate the rank of the matrix, $\mathbf{A}$. If $\mathbf{A}$ is not a full column rank matrix, the new sampling point is removed and the sampled set and candidate set are updated. To achieve the highest accuracy, the operation of sampling must be repeated until the number of retained sampling points in the sampled set is greater than the number of expansion coefficients. Generally, the number of sampling points is 1.0-1.3 times of $N_{c}$. The new sampling points can still be selected by minimizing $\Phi_{q}$.

3.4. Procedure of the SGS-IRAPC Method. This study proposes a new surrogate model which combined the IRMAPC expansion method with a novel sequential sampling scheme. The proposed method can improve the computational efficiency for multidimensional problems with interval and random uncertain variables by reducing the number of sampling points used to determine the expansion coefficient of the IRMAPC expansion method. The proposed method is called the SGS-IRAPC expansion method. As summarized, the detailed procedures of the SGS-IRAPC expansion method are as follows:

Step 1. Calculate the polynomial basis, Gaussian integration points, and Gaussian weights for each variable according to $[37,45]$

Step 2. Obtain the integration points in the sparse grids' quadrature by (13)-(16)

Step 3. Obtain the candidate set through (16) and homogenize the candidate set

Step 4. Determine the first sampling point and rank the sampling points of the candidate set through (17) and (18)

Step 5. Determine the initial sampled set uniformly

Step 6. Select more sampling points from the candidate set by minimizing (19), and delete the points in the sampling set that cause the matrix, $\mathbf{A}$, in (10) to be not a full column rank matrix

Step 7. Repeated Step 6 until the number of the sampling points is denoted as 1.0-1.3 times of $N_{c}$

Step 8. Calculate the expansion coefficient through (10)-(12)

Step 9. Calculate the function, $F(x)$, with multidimensional variables through $(7)$

\section{SGS-IRAPC for Vibro-Acoustic Analysis with Interval and Random Uncertainties}

4.1. Vibro-Acoustic Systems with Interval and Random Uncertainties. The acoustic finite element analysis [28] is used to establish the dynamic equilibrium equation of the vibro-acoustic system [28]. Under the time harmonic excitation, the dynamic equilibrium equation of the vibroacoustic system can be given as the following matrix form: 


$$
\left[\begin{array}{cc}
\mathbf{K}_{v s}-\omega^{2} \mathbf{M}_{v s} & -\mathbf{H} \\
\rho \omega^{2} \mathbf{H}^{T} & \mathbf{K}_{a c}-\omega^{2} \mathbf{M}_{a c}
\end{array}\right]\left\{\begin{array}{c}
\mathbf{u}_{v s} \\
\mathbf{P}
\end{array}\right\}=\left\{\begin{array}{l}
\mathbf{F}_{v s} \\
\mathbf{F}_{a c}
\end{array}\right\},
$$

where $\omega$ represents the angular frequency of the time harmonic, $\rho$ represents the density of the acoustic fluid, $\mathbf{K}_{v s}$ and $\mathbf{M}_{v s}$ represent the structural stiffness matrix and the mass matrix, respectively, $\mathbf{K}_{a c}$ and $\mathbf{M}_{a c}$ represent the acoustic stiffness matrix and the mass matrix, respectively, $\mathbf{H}$ denotes the spatial coupled matrix, $\mathbf{u}_{v s}$ is the structure displacement vector, $\mathbf{P}$ denotes the sound pressure vector in the acoustic cavity, and $\mathbf{F}_{v s}$ and $\mathbf{F}_{a f}$ denote the generalized force vectors acting on the vibrating structure and the acoustic cavity, respectively.

By simplifying, dynamic equilibrium equation (23) can be denoted as the following formula:

$$
\mathbf{Z R}=\mathbf{F} .
$$

In (24),

$$
\begin{aligned}
& \mathbf{Z}=\left[\begin{array}{cc}
\mathbf{K}_{v}-\omega^{2} M_{v s} & -\mathbf{H} \\
\rho \omega^{2} \mathbf{H}^{T} & \mathbf{K}_{a c}-\omega^{2} \mathbf{M}_{a c}
\end{array}\right], \\
& \mathbf{R}=\left\{\begin{array}{c}
\mathbf{u}_{v s} \\
\mathbf{P}
\end{array}\right\}, \\
& \mathbf{F}=\left\{\begin{array}{c}
\mathbf{F}_{v s} \\
\mathbf{F}_{a c}
\end{array}\right\},
\end{aligned}
$$

where $\mathbf{F}$ represents the external excitation vector of the vibro-acoustic system, $\mathrm{Z}$ represents the vibro-acoustic dynamic stiffness matrix, and $\mathbf{R}$ represents the vibro-acoustic response vector.

The uncertainties widely exist in the practical vibroacoustic system due to the effect of the multipurpose characteristics of materials and the unpredictability of the environment. The vibro-acoustic system dynamic equilibrium equation can be given by representing the uncertain parameters by a vector, $x$, as follows:

$$
\mathbf{Z}(\mathbf{x}) \mathbf{R}(\mathbf{x})=\mathbf{F}(\mathbf{x}) \text {. }
$$

In this study, the uncertain parameters, whose random moments or PDFs are available, are considered as random variables and are represented by a vector, $\mathbf{x}^{R}$. On the contrary, the uncertain parameters with incomplete random data are considered as interval variables and are represented by a vector, $\mathbf{x}^{I}$. As the applications of practical engineering applications are complex, the interval and random uncertain parameters may be included simultaneously. In the complex vibro-acoustic system, some uncertain parameters are considered as random variables, and the other uncertain part is considered as interval variables. Thus, all the uncertain parameters of the vibro-acoustic system can be denoted as $\mathbf{x}=\left[\mathbf{x}^{R}, \mathbf{x}^{I}\right]$.
4.2. The Interval and Random Analysis of Uncertain Vibro-Acoustic System. The uncertain problems with interval and random variables calculated by the SGS-IRAPC can be expressed as equation (3). The interval and random analysis of an uncertain vibro-acoustic system requires two steps. Primary, the interval variable is assumed as constant parameters. Based on this assumption, equation (3) can be represented as follows:

$$
\begin{aligned}
& F(x)=\sum_{i_{1}=0}^{N_{1}} \ldots \sum_{i_{L_{2}}=0}^{N_{L_{2}}}\left(\sum_{i_{1}=0}^{N_{1}} \ldots \sum_{i_{L_{1}}=0}^{N_{L_{1}}} f_{i_{1} \ldots i_{L}} G_{i_{1} \ldots i_{L_{1}}}\left(\mathbf{x}^{I}\right)\right) G_{i_{1}, \ldots, i_{L_{2}}}\left(\mathbf{x}^{R}\right), \\
& F(x)=\sum_{i_{1}=0}^{N_{L_{R}}} \ldots \sum_{i_{L}=0}^{N_{L_{R}}} z_{i_{1} \ldots i_{L_{R}}} G_{i_{1} \ldots i_{L_{R}}}\left(\mathbf{x}^{R}\right),
\end{aligned}
$$

where

$$
z_{i_{L_{1}} \ldots i_{L_{R}}}=\sum_{i_{1}=0}^{N_{1}} \ldots \sum_{i_{L_{1}}=0}^{N_{L_{1}}} f_{i_{1}, \ldots, i_{L}} G_{i_{1}, \ldots, i_{L_{1}}}\left(\mathbf{x}^{I}\right) .
$$

Based on the orthogonality of the SGS-IRAPC, the expectation is represented as

$$
\begin{aligned}
\mu & =E\left[\sum_{i_{1}=0}^{N_{1}} \ldots \sum_{i_{L_{2}}=0}^{N_{L_{2}}} z_{i_{1} \cdots i_{L_{2}}} G_{i_{1} \cdots i_{L_{2}}}\left(\mathbf{x}^{R}\right)\right] \\
& =z_{0, \ldots, 0 .} .
\end{aligned}
$$

In equation (29), $\omega_{j}\left(x_{j}^{R}\right)\left(j=1 \ldots L_{R}\right)$ is the weight function of the polynomial basis related to $x_{j}^{R}$.

Similarly, $E\left[F(x)^{2}\right]$ can be expressed as

$$
\begin{aligned}
E\left[F(x)^{2}\right] & =E\left[\left(\sum_{i_{1}=0}^{N_{1}} \ldots \sum_{i_{L_{2}}=0}^{N_{L_{2}}} z_{i_{1} \ldots i_{L_{2}}} G_{i_{1} \ldots i_{L_{2}}}\left(\mathbf{x}^{R}\right)\right)^{2}\right] \\
& =\sum_{i_{1}=0}^{N_{1}} \ldots \sum_{i_{L_{2}}=0}^{N_{L_{2}}}\left(z_{i_{1} \ldots i_{L_{2}}}\right)^{2} .
\end{aligned}
$$

According to equations (29) and (30), the variance of the response can be expressed as follows:

$$
\sigma^{2}(\mathbf{x})=E\left[\left(\sum_{i_{1}=0}^{N_{1}} \ldots \sum_{i_{L_{2}}=0}^{N_{L_{2}}} z_{i_{1} \ldots i_{L_{2}}} G_{i_{1} \ldots i_{L_{2}}}\left(\mathbf{x}^{R}\right)\right)^{2}\right]-\left(z_{0, \ldots, 0}\right)^{2} .
$$

Substituting equation (28) into equations (30) and (31), the expectation and the variance of the response of the uncertain vibro-acoustic system can be rewritten as 


$$
\begin{aligned}
\mu\left(\mathbf{x}^{I}\right) & =\sum_{i_{1}=0}^{N_{1}} \ldots \sum_{i_{L_{1}}=0}^{N_{L_{1}}} f_{i_{1}, \ldots, i_{L_{1}}, 0, \ldots, 0} G_{i_{1}, \ldots, i_{L_{1}}}\left(\mathbf{x}^{I}\right), \\
\sigma^{2}\left(\mathbf{x}^{I}\right) & =\sum_{i_{1}=0}^{N_{1}} \ldots \sum_{i_{L_{2}}=0}^{N_{L_{2}}}\left(\sum_{i_{1}=0}^{N_{1}} \ldots \sum_{i_{L_{1}}=0}^{N_{L_{1}}} f_{i_{1}, \ldots, i_{L}} G_{i_{1}, \ldots, i_{L_{1}}}\left(\mathbf{x}^{I}\right)\right)^{2}-\left(\sum_{i_{1}=0}^{N_{1}} \ldots \sum_{i_{L_{1}}=0}^{N_{L_{1}}} f_{i_{1}, \ldots, i_{L_{1}}, 0, \ldots, 0} G_{i_{1}, \ldots, i_{L_{1}}}\left(\mathbf{x}^{I}\right)\right)^{2} .
\end{aligned}
$$

In the last step, the bounds of the expectation and the variance of an uncertain vibro-acoustic analysis can be calculated by the MC method, which can be expressed as follows:

$$
\left[\underline{\sigma^{2}}, \overline{\sigma^{2}}\right] \equiv\left[\min _{x_{j}^{I} \in[\underline{x}, \bar{x}]}\left\{\sigma^{2}\left(\mathbf{x}^{I}\right)\right\}, \max _{x_{j}^{I} \in[\underline{x}, \bar{x}]}\left\{\sigma^{2}\left(\mathbf{x}^{I}\right)\right\}\right],[\underline{\mu}, \bar{\mu}]=\left[\min _{x_{j}^{I} \in[\underline{x}, \bar{x}]}\left\{\mu\left(\mathbf{x}^{I}\right)\right\}, \max _{x_{j}^{I} \in[\underline{x}, \bar{x}]}\left\{\mu\left(\mathbf{x}^{I}\right)\right\}\right] .
$$

\section{Numerical Examples}

The SGS-IRAPC is applied to a simple mathematical function and a vibro-acoustic problem to analyze its performance in this section. The calculation results are compared to those of the Sequential Sampling Scheme-based Interval and Random Arbitrary Polynomial Chaos (SSSIRAPC) [43] and the IRMAPC [37].

5.1. Simple Mathematical Problem. In this numerical example, a simple function with five random variables and one interval variable is considered as follows:

$$
y(x)=e^{0.25\left(x_{1}+x_{2}+\cdots+x_{6}\right)},
$$

where $x_{6}$ represents the interval variable and $x_{i}(i=1,2, \ldots, 5)$ represents random variable. Only statistical data is available for the random variable in this case. The range of each variable is $[-1,1]$. Figure 3 shows the frequency distribution histogram of the random variables.

To analyze the accuracy of the SGS-IRAPC, the relative error of expectation and variance is defined as follows:

$$
\begin{aligned}
& e r_{\mu}=\max \left\{\left|\frac{\bar{\mu}-\bar{\mu}_{\mathrm{ref}}}{\bar{\mu}_{\mathrm{ref}}}\right|,\left|\frac{\underline{\underline{\mu}-\underline{\mu}}}{\underline{\underline{\mu}}-\underline{\mathrm{ref}}_{\mathrm{ref}}}\right|\right\}, \\
& e r_{\sigma}=\max \left\{\left|\frac{\bar{\sigma}^{2}-\bar{\sigma}_{\mathrm{ref}}^{2}}{\bar{\sigma}_{\mathrm{ref}}^{2}}\right|,\left|\frac{\underline{\sigma}-\underline{\sigma}_{\mathrm{ref}}^{2}}{\underline{\sigma}_{\mathrm{ref}}^{2}}\right|\right\} .
\end{aligned}
$$

In equations (35) and (36), $\bar{\sigma}_{\text {ref }}^{2}, \underline{\sigma}_{\text {ref }}^{2}, \bar{\mu}_{\text {ref }}$, and $\underline{\mu}_{\text {ref }}$ denote the reference results which are acquired from the $\mathrm{MC}$ method. In the MC method, the number of sampling points for the random variables is 5000 , and for the interval variable, it is $20 . e r_{\mu}$ and $e r_{\sigma}$ of the different methods are shown in Figure 4.

By comparing the results obtained from the SGS-IRAPC and the SSS-IRAPC in Figure 4, it is observed that $e r_{\sigma}$ and $e r_{\mu}$ obtained from the SGS-IRAPC are smaller than those obtained from the SSS-IRAPC. This is due to the errors introduced by the SSS-IRAPC in the estimation of PDFs of random variables, while the MAPC method can construct the polynomial basis from the moment of the random variables. Essentially, the SGS-IRAPC and IRMAPC can prevent the errors due to the estimation of PDFs. Therefore, the SGS-IRAPC can achieve greater accuracy than the SSSIRAPC.

When comparing the convergence properties of the SGSIRAPC and the IRMAPC, it is observed from Figure 4 that $e r_{\sigma}$ and $e r_{\mu}$ obtained from the SGS-IRAPC are smaller than those obtained from the IRMAPC with the same sampling points. This indicates that the IRMAPC requires more sampling points to achieve the same level of precision. The main difference between the SGS-IRAPC and the IRMAPC is that the coefficient of the SGS-IRAPC is calculated by using the proposed sparse grids' sequential sampling scheme instead of the Gaussian quadrature in the IRMAPC. It observed that the application of the sparse grids' sequential sampling scheme significantly decreases the computational cost of the arbitrarily polynomial chaos method for the interval and random analysis.

To compare the computational cost of SGS-IRAPC and SSS-IRAPC more clearly, the time periods for the calculation of the expansion coefficients and the total computational time of the SGS-IRAPC and SSS-IRAPC at the same orders using Matlab R2018a on a $1.80 \mathrm{GHz}$ Intel(R) Core(TM) i7$8565 \mathrm{U}$ CPU are listed in Table 3.

As illustrated in Table 3, the retained order is 5 and the total computational time periods of the SGS-IRAPC and the SSS-IRAPC are $84.1469 \mathrm{~s}$ and $736.2311 \mathrm{~s}$, respectively. This indicates that the SGS-IRAPC presents a significant increase of computing efficiency when compared to the SSS-IRAPC. This is because the number of integration points in the candidate set used for the sequential sampling scheme of the SGS-IRAPC is substantially reduced by the sparse grids' scheme which is based on the Smolyak algorithm. Thus, the SGS-IRAPC exhibits superior accuracy and also achieves a significant increase of computing efficiency for the interval and random analysis.

5.2. Vibro-Acoustic System Related to Hybrid Interval and Random Uncertainties. The shell vibro-acoustic system related to hybrid interval and random uncertainties is analyzed 
$\left(x_{1}\right)$

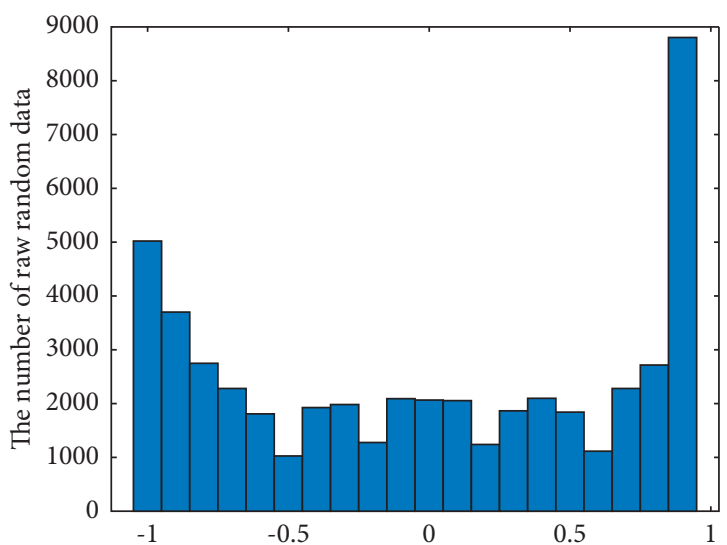

$\left(x_{3}\right)$

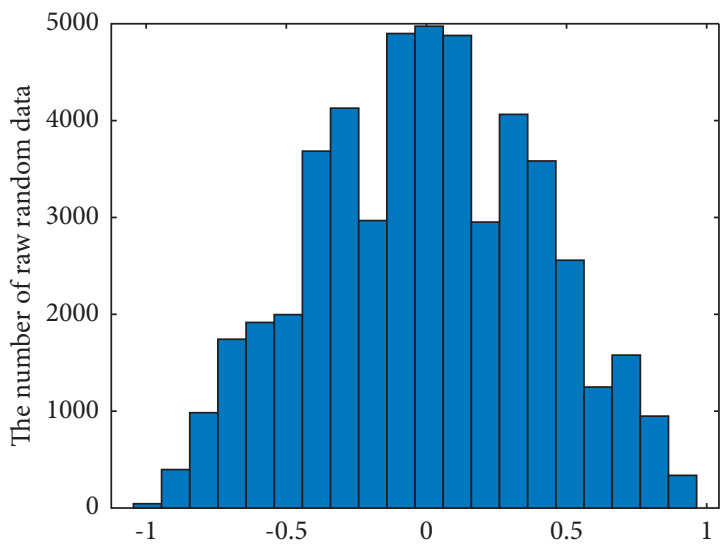

$\left(x_{2}\right)$

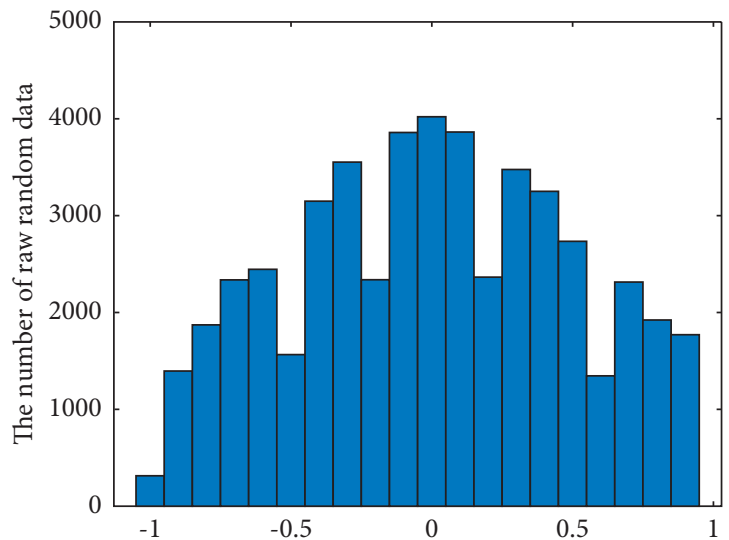

$\left(x_{4} / x_{5}\right)$

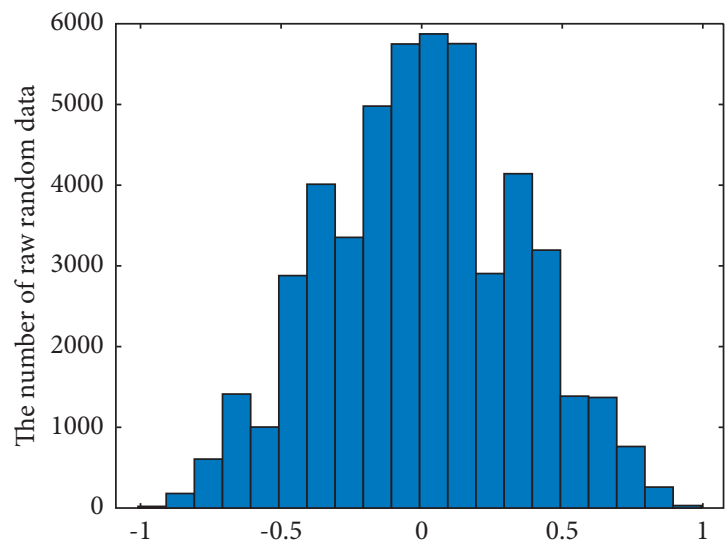

FIGURE 3: Frequency distribution histogram of random variables.

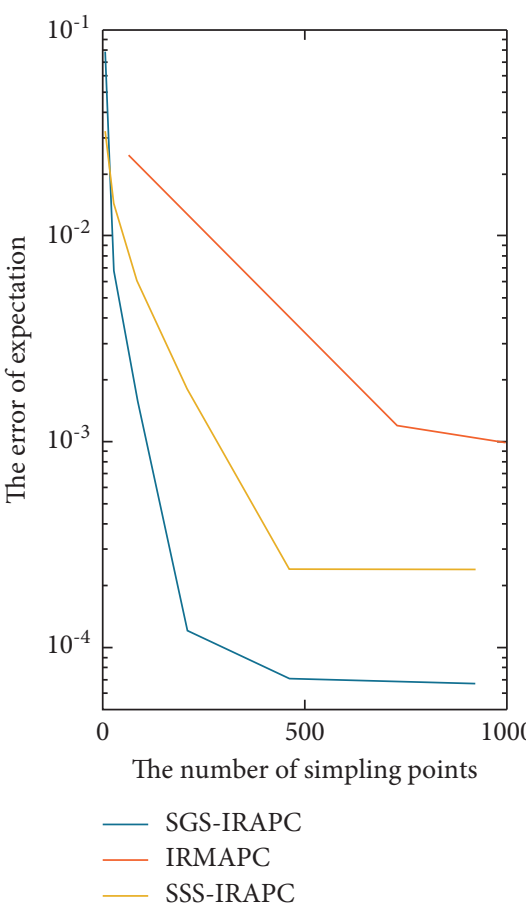

(a)

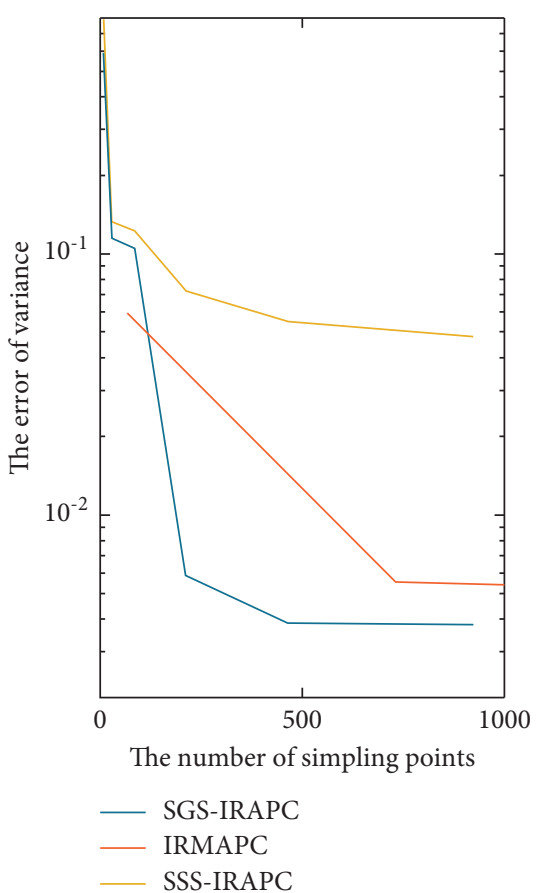

(b)

FIGURE 4: Calculated $e r_{\mu}$ and $e r_{\sigma}$ using SGS-IRAPC, SSS-IRAPC, and IRMAPC at different sampling points: (a) expectation; (b) variance. 
TABLE 3: Time periods to calculate the expansion coefficients, and the total execution time of SGS-IRAPC and SSS-IRAPC at the same orders.

\begin{tabular}{lccc}
\hline Order & Method & Time for calculation of the expansion coefficient (s) & Total computational time (s) \\
\hline \multirow{2}{*}{1} & SSS-IRAPC & 0.1872 & 0.2496 \\
& The proposed SGS-IRAPC & 1.9032 & 1.9500 \\
\hline \multirow{2}{*}{2} & SSS-IRAPC & 0.8580 & 0.9672 \\
& The proposed SGS-IRAPC & 4.8828 & 4.9296 \\
\hline \multirow{2}{*}{4} & SSS-IRAPC & 13.1041 & 13.2913 \\
& The proposed SGS-IRAPC & 11.9185 & 12.0589 \\
\hline \multirow{2}{*}{5} & SSS-IRAPC & 295.1695 & 295.7155 \\
& The proposed SGS-IRAPC & 83.6009 & 84.0845 \\
\hline
\end{tabular}

TABLE 4: Uncertain parameters of the uncertain shell vibro-acoustic systems in this section.

\begin{tabular}{lc}
\hline Uncertain parameters & Case \\
\hline$t(\mathrm{~mm})$ & $3+0.9 x_{1}^{R}$ \\
$c(\mathrm{~m} / \mathrm{s})$ & $340+17 x_{2}^{R}$ \\
$E(\mathrm{GPa})$ & $210+63 x_{3}^{R}$ \\
$v$ & $0.3+0.09 x_{4}^{R}$ \\
$\rho_{s}\left(\mathrm{~kg} / \mathrm{m}^{3}\right)$ & $7850+2355 x_{5}^{R}$ \\
$\rho_{f}\left(\mathrm{~kg} / \mathrm{m}^{3}\right)$ & {$[0.96-1.44]$} \\
\hline
\end{tabular}
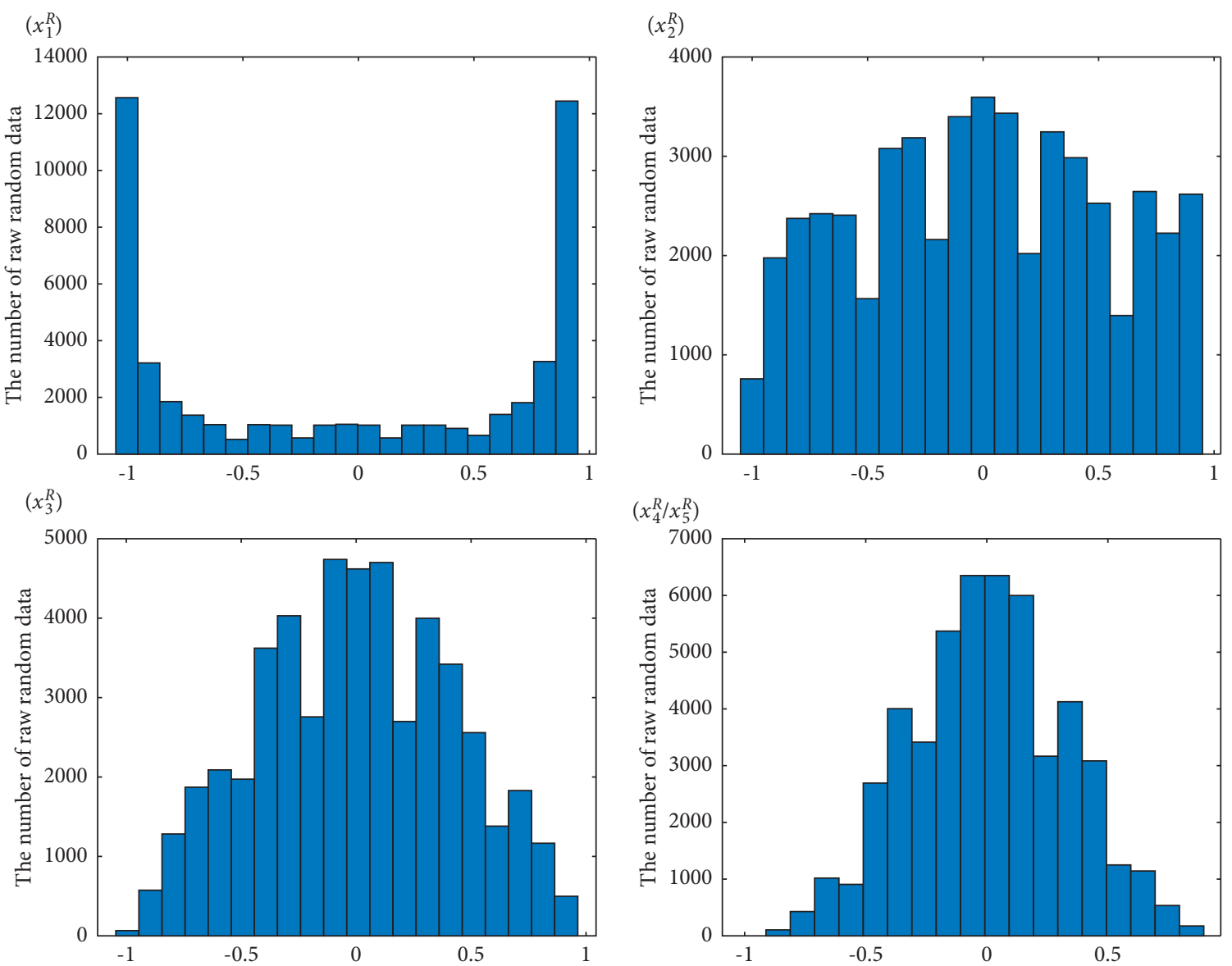

FiguRE 5: Frequency distribution histogram of random variables. 


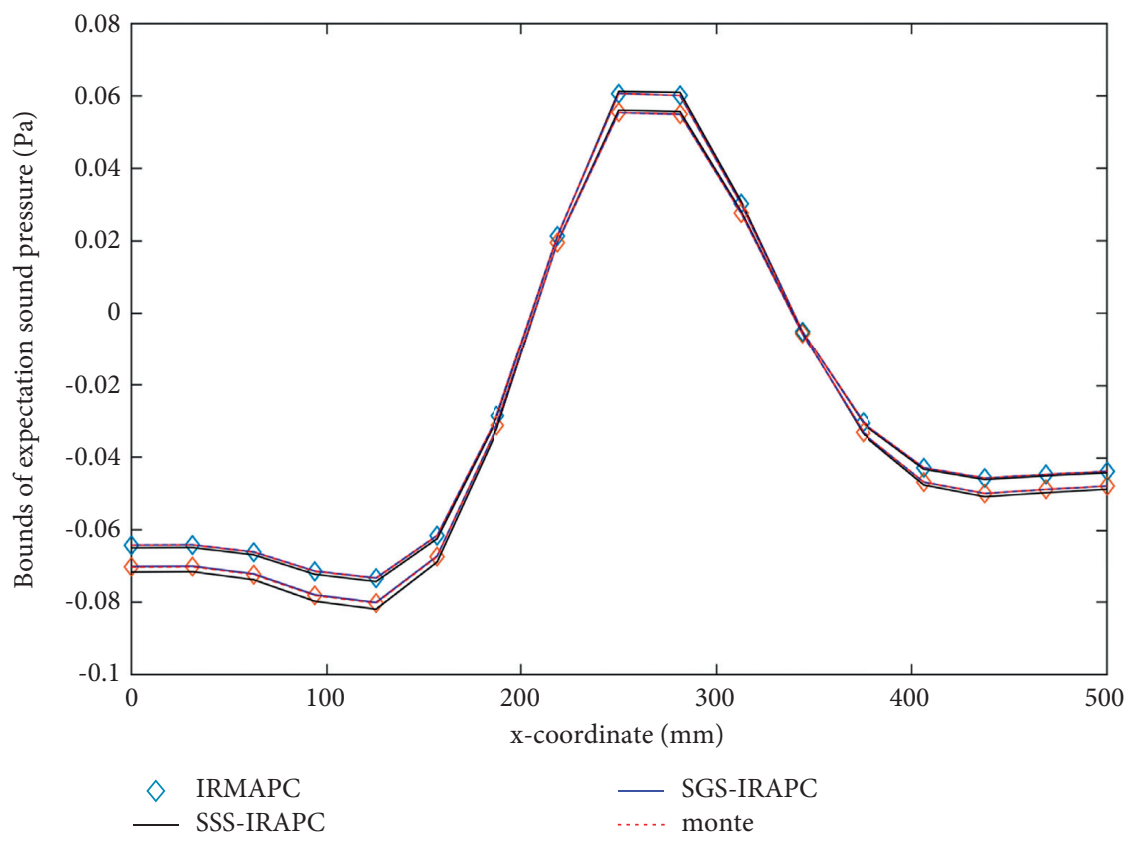

(a)

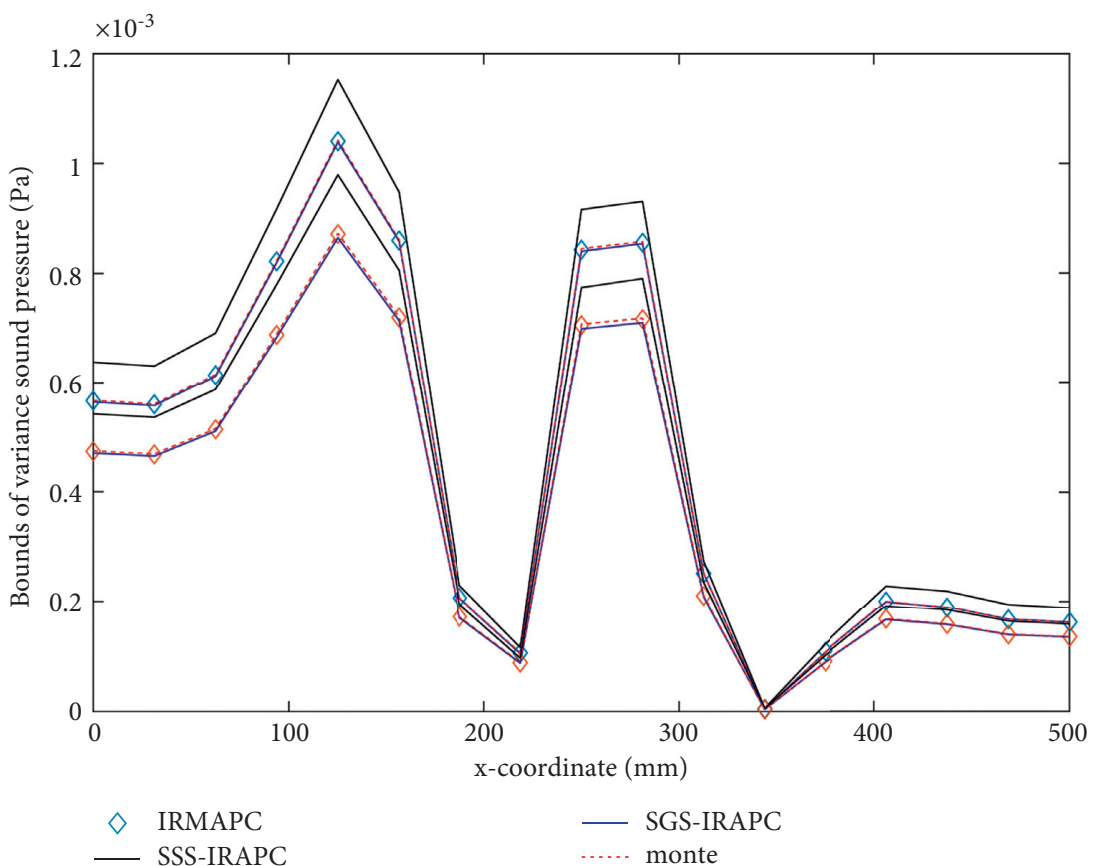

(b)

FIGURE 6: Bounds of expectation and variance of the sound pressure distributing in the middle section for $f=200 \mathrm{~Hz}$ obtained from the SSS-IRAPC, IRMAPC, and SGS-IRAPC. (a) Expectation. (b) Variance.

in this section using the finite element. The shell is made of steel, with the parameters $\rho_{s}=7850 \mathrm{~kg} / \mathrm{m}^{3}, E=2.1 \times 10^{5}$ $\mathrm{MPa}$, and $v=0.3$. The thickness of the shell is $3 \mathrm{~mm}$. The acoustic cavity in this study is filled with air $\left(\rho_{f}=1.2 \mathrm{~kg} / \mathrm{m}^{3}\right.$ and $\left.c=340 \mathrm{~m} / \mathrm{s}\right)$. The shell is located at the top of the vibro-acoustic system, and all its shell are fixed, while the walls of the acoustic cavity are rigid. The load vector in the $Z$-direction is loaded at the center point of the shell, and its magnitude is $1 \mathrm{~N}$.
Due to the unpredictability of the temperature of the environment and errors in the manufacturing process of the materials, $v, \rho_{f}, c, \rho_{s}, E$, and thickness, $t$, are regarded as independent uncertain parameters. All the uncertain parameters are assumed as the linear function of the uncertain variables; the uncertainty information of this case is illustrated as Table 4.

The range of the interval variable is $[0.96,-1.44]$. The statistical data for random variables is available, which 
TABLE 5: Computational time of the IRMAPC, the SSS-IRAPC, and the SGS-IRAPC.

\begin{tabular}{lccc}
\hline Uncertain models & IRMAPC & SSS-IRAPC & The proposed SGS-IRAPC \\
\hline Computational time (s) & $5.68 \times 10^{11}$ & $6.72 \times 10^{9}$ & $3.25 \times 10^{9}$ \\
Response time (s) & $5.68 \times 10^{11}$ & $6.64 \times 10^{9}$ & $2.97 \times 10^{9}$ \\
\hline
\end{tabular}

ranges from $[-1,1]$. For conciseness, the frequency distribution histogram of the statistical data of random variables is shown in Figure 5.

In this section, the SGS-IRAPC, the IRMAPC, and the SSS-IRAPC are used to analyse the response analysis of the vibro-acoustic problem. In this section, the orders of the arbitrary polynomial chaos expansion are 3 for all the uncertain variables $\left(t, E, c, \rho_{s}, v\right.$, and $\left.\rho_{f}\right)$. The solution of the MC method is considered as reference in this section, and the number of sampling points for the random variable and the interval variable is 50,000 and 20 , respectively. The bounds of expectation and variance of the sound pressure distributed in the middle section for $f=200 \mathrm{~Hz}$ yielded by the SGS-IRAPC, the IRMAPC, the SSS-IRAPC, and the MC method are shown in Figure 6.

The lower and upper bounds of the expectation and variance of the sound pressure distributed in the middle section obtained from the proposed methods and the other arbitrarily polynomial chaos methods are plotted in Figure 6. It can be obviously observed that the expectation and the variance of the sound pressure yielded by the IRMAPC and SGS-IRAPC are much closer to the reference results than those obtained by the SSS-IRAPC. In other words, the computing precision of the SGS-IRAPC is close to that of the IRMAPC and much higher than that of the SSS-IRAPC.

The computational time and the sampling time of the IRMAPC, the SSS-IRAPC, and the SGS-IRAPC are obtained using Matlab R2018a on a $3.30 \mathrm{GHz}$ Intel(R) Core(TM) i910900k CPU, as shown in Table 5. It is observed that the computational time obtained from the SGS-IRAPC is much shorter than that of the IRMAPC and slightly shorter than that of the SSS-IRAPC. The SGS-IRAPC presents a much higher efficiency when compared to the IRMAPC and the SSS-IRAPC. It indicates that the SGS-IRAPC can greatly improve the computing efficiency without affecting the precision and it is more suitable than the IRMAPC and the SSS-IRAPC for the interval and random analysis of an uncertain vibro-acoustic system with multidimension uncertainty.

\section{Conclusion}

A novel sparse polynomial expansion method is proposed in this study to evaluate the interval and random uncertain problems efficiently and effectively. The method is called the Sparse Grids' Sequential Sampling-based Interval and Random Arbitrary Polynomial Chaos (SGS-IRAPC). In the SGS-IRAPC, the response of the interval and random mixed uncertainties model is approximated by the MAPC method, which can construct the polynomial basis for the random variable without determined PDF of the random variable. Additionally, a sparse sequential sampling scheme is proposed in the SGS-IRAPC to decrease the computational cost for the response of the uncertain vibro-acoustic system with both interval and random variables. The sparse grids' scheme is combined with the sequential sampling scheme in this method.

The following are the advantages provided by the proposed method. For multidimensional problems, the SGS-IRAPC greatly improves the computational efficiency without affecting the precision. To improve the computing efficiency, the SGS-IRAPC method selects a part of the integration points in the candidate set, as the required points with the help of the sequential sampling scheme. In addition, to reduce the number of the integration points in the candidate set, the candidate set is obtained from the sparse grids' scheme which is based on the Smolyak algorithm. This process further improves the computing efficiency. Thus, the proposed SGS-IRAPC method sharply improves the efficiency when compared to the traditional arbitrarily polynomial chaos expansion method.

A mathematical function and a vibro-acoustic problem with both interval and random uncertainties are introduced to embody the effectiveness of the SGS-IRAPC. The merits of the SGS-IRAPC are demonstrated by comparing it with the SSS-IRAPC and the IRMAPC. The numerical results demonstrate that the SGS-IRAPC greatly improves the calculation efficiency without affecting the precision. Therefore, the SGS-IRAPC can be considered as a convenient and effective method for the response analysis of the vibroacoustic system related to both interval and random uncertainties.

\section{Data Availability}

The data used to support the findings of this study are included within the article.

\section{Conflicts of Interest}

The authors declare that there are no conflicts of interest regarding the publication of this article.

\section{Acknowledgments}

The study was supported by the Natural Science Foundation of Hunan Province, China (no. 2020JJ5686).

\section{References}

[1] D. J. Nefske, J. A. Wolf, and L. J. Howell, "Structural-acoustic finite element analysis of the automobile passenger compartment: a review of current practice," Journal of Sound and Vibration, vol. 80, no. 2, pp. 247-266, 1982.

[2] A. Seçgin, J. F. Dunne, and L. Zoghaib, "Extreme-value-based statistical bounding of low, mid, and high frequency responses 
of a forced plate with random boundary conditions," Journal of Vibration and Acoustics, vol. 134, no. 2, 2012.

[3] R. E. Caflisch, "Monte Carlo and quasi-Monte Carlo methods," Acta Numerica, vol. 7, no. 2, pp. 1-49, 1998.

[4] M. N. Ichchou, F. Bouchoucha, M. A. S. Ben, O. Dessombz, and M. Haddar, "Stochastic wave finite element for random periodic media through first-order perturbation," Computer Methods in Applied Mechanics and Engineering, vol. 200, no. 41-44, pp. 2805-2813, 2011.

[5] M. Kaminski, "Stochastic second-order perturbation approach to the stress-based finite element method," International Journal of Solids and Structures, vol. 38, no. 21, pp. 3831-3852, 2001.

[6] I. Doltsinis and Z. Kang, "Perturbation-based stochastic FE analysis and robust design of inelastic deformation processes," Computer Methods in Applied Mechanics and Engineering, vol. 195, no. 19-22, pp. 2231-2251, 2006.

[7] X. G. Hua, Y. Q. Ni, Z. Q. Chen, and J. M. Ko, “An improved perturbation method for stochastic finite element model updating," International Journal for Numerical Methods in Engineering, vol. 73, no. 13, pp. 1845-1864, 2008.

[8] R. G. Ghanem and P. Spanos, Stochastic Finite Elements: A Spectral Approach, Springer-Verlag, New York, NY, USA, 1991.

[9] D. Xiu and G. E. Karniadakis, "Modeling uncertainty in flow simulations via generalized polynomial chaos," Journal of Computational Physics, vol. 187, no. 1, pp. 137-167, 2003.

[10] D. Xiu and G. E. Karniadakis, "The wa polynomial chaos for stochastic differential equations," SIAM Journal on Scientific Computing, vol. 24, no. 2, pp. 619-644, 2002.

[11] S. Rao and L. Berke, "Analysis of uncertain structural systems using interval analysis,” AIAA Journal, vol. 35, no. 4, pp. 727-735, 1997.

[12] Z. Qiu and I. Elishakoff, "Antioptimization of structures with large uncertain-but-non-random parameters via interval analysis," Computer Methods in Applied Mechanics and Engineering, vol. 152, no. 3-4, pp. 361-372, 1998.

[13] I. P. Gavrilyuk, "Book review: introduction to interval analysis," Mathematics of Computation, vol. 79, no. 269, p. 615, 2010.

[14] H. Yin, D. Yu, S. Yin, and B. Xia, "Fuzzy interval Finite Element/Statistical Energy Analysis for mid-frequency analysis of built-up systems with mixed fuzzy and interval parameters," Journal of Sound and Vibration, vol. 380, pp. 192-212, 2016.

[15] D. Moens and D. Vandepitte, "A fuzzy finite element procedure for the calculation of uncertain frequency-response functions of damped structures: Part 1-Procedure," Journal of Sound and Vibration, vol. 288, no. 3, pp. 431-462, 2005.

[16] H.-R. Bae, R. V. Grandhi, and R. A. Canfield, "Epistemic uncertainty quantification techniques including evidence theory for large-scale structures," Computers \& Structures, vol. 82, no. 13-14, pp. 1101-1112, 2004.

[17] C. Jiang, Z. Zhang, X. Han, and J. Liu, "A novel evidencetheory-based reliability analysis method for structures with epistemic uncertainty," Computers \& Structures, vol. 129, pp. 1-12, 2013.

[18] S. Yin, D. Yu, H. Yin, and B. Xia, "A new evidence-theorybased method for response analysis of acoustic system with epistemic uncertainty by using Jacobi expansion," Computer Methods in Applied Mechanics and Engineering, vol. 322, no. 1, pp. 419-440, 2017.

[19] N. Chen, D. Yu, B. Xia, and M. Beer, "Uncertainty analysis of a structural-acoustic problem using imprecise probabilities based on p-box representations," Mechanical Systems and Signal Processing, vol. 80, pp. 45-57, 2016.

[20] C. Simon and F. Bicking, "Hybrid computation of uncertainty in reliability analysis with p-box and evidential networks," Reliability Engineering \& System Safety, vol. 167, pp. 629-638, 2017.

[21] J. Wu, Y. Zhang, L. Chen, and Z. Luo, "A Chebyshev interval method for nonlinear dynamic systems under uncertainty," Applied Mathematical Modelling, vol. 37, no. 6, pp. 4578-4591, 2013.

[22] Z. Qiu, L. Ma, and X. Wang, "Non-probabilistic interval analysis method for dynamic response analysis of nonlinear systems with uncertainty," Journal of Sound and Vibration, vol. 319, no. 1-2, pp. 531-540, 2009.

[23] B. Xia and D. Yu, "Modified sub-interval perturbation finite element method for 2D acoustic field prediction with large uncertain-but-bounded parameters," Journal of Sound and Vibration, vol. 331, no. 16, pp. 3774-3790, 2012.

[24] J. Wu, Z. Luo, Y. Zhang, N. Zhang, and L. Chen, "Interval uncertain method for multibody mechanical systems using Chebyshev inclusion functions," International Journal for $\mathrm{Nu}$ merical Methods in Engineering, vol. 95, no. 7, pp. 608-630, 2013.

[25] J. Wu, Z. Luo, N. Zhang, and Y. Zhang, "A new uncertain analysis method and its application in vehicle dynamics," Mechanical Systems and Signal Processing, vol. 51, pp. 659-675, 2015.

[26] B. Xia, Y. Qin, D. Yu, and C. Jiang, "Dynamic response analysis of structure under time-variant interval process model," Journal of Sound and Vibration, vol. 381, pp. 121-138, 2016.

[27] C. Li, B. Chen, H. Peng, and S. Zhang, "Sparse regression Chebyshev polynomial interval method for nonlinear dynamic systems under uncertainty," Applied Mathematical Modelling, vol. 51, pp. 505-525, 2017.

[28] W. Gao, "Interval natural frequency and mode shape analysis for truss structures with interval parameters," Finite Elements in Analysis and Design, vol. 42, no. 6, pp. 471-477, 2006.

[29] Z. Qiu, Y. Xia, and J. Yang, "The static displacement and the stress analysis of structures with bounded uncertainties using the vertex solution theorem," Computer Methods in Applied Mechanics and Engineering, vol. 196, no. 49-52, pp. 49654984, 2007.

[30] S. Yin, D. Yu, Z. Ma, and B. Xia, "A unified model approach for probability response analysis of structure-acoustic system with random and epistemic uncertainties," Mechanical Systems and Signal Processing, vol. 111, pp. 509-528, 2018.

[31] W. A. Lodwick and K. D. Jamison, "Interval-valued probability in the analysis of problems containing a mixture of possibilistic, probabilistic, and interval uncertainty," Fuzzy Sets and Systems, vol. 159, no. 21, pp. 2845-2858, 2008.

[32] C. Wang and H. G. Matthies, "Hybrid evidence-and-fuzzy uncertainty propagation under a dual-level analysis framework," Fuzzy Sets and Systems, vol. 367, pp. 51-67, 2019.

[33] H. Lü, Z. Cai, Q. Feng, W. B. Shangguan, and D. Yu, “An improved method for fuzzy-interval uncertainty analysis and its application in brake instability study," Computer Methods in Applied Mechanics and Engineering, vol. 342, pp. 142-160, 2018.

[34] I. Elishakoff and P. Colombi, "Combination of probabilistic and convex models of uncertainty when scarce knowledge is present on acoustic excitation parameters," Computer Methods in Applied Mechanics and Engineering, vol. 104, no. 2, pp. 187-209, 1993. 
[35] S. Yin, D. Yu, H. Yin, and B. Xia, "Interval and random analysis for structure-acoustic systems with large uncertainbut-bounded parameters," Computer Methods in Applied Mechanics and Engineering, vol. 305, pp. 910-935, 2016.

[36] S. Yin, D. Yu, Z. Luo, and B. Xia, "Unified polynomial expansion for interval and random response analysis of uncertain structure-acoustic system with arbitrary probability distribution," Computer Methods in Applied Mechanics and Engineering, vol. 336, no. 1, pp. 260-285, 2018.

[37] N. Chen, J. Chen, and S. Yin, "Moment-based hybrid polynomial chaos method for interval and random uncertain analysis of periodical composite structural-acoustic system with multi-scale parameters," International Journal of Computational Methods, vol. 18, no. 4, 2020.

[38] M. Thapa, S. B. Mulani, and R. W. Walters, "Adaptive weighted least-squares polynomial chaos expansion with basis adaptivity and sequential adaptive sampling," Computer Methods in Applied Mechanics and Engineering, vol. 360, Article ID 112759, 2020.

[39] M. D. McKay, R. J. Beckman, and W. J. Conover, "A comparison of three methods for selecting values of input variables in the analysis of output from a computer code," Technometrics, vol. 42, no. 1, pp. 55-61, 2000.

[40] A. Owen, "Orthogonal arrays for computer experiments, integration and visualization," Statistica Sinica, vol. 2, no. 2, pp. 439-452, 1992.

[41] D. C. Montgomery, Design and Analysis of Experiments, Post \& Telecom Press, Beijing, China, 6th edition, 2006.

[42] Z. Liu, D. Lesselier, B. Sudret, and J. Wiart, "Surrogate modeling based on resampled polynomial chaos expansions," Reliability Engineering \& System Safety, vol. 202, Article ID 107008, 2020.

[43] J. Wu, Z. Luo, J. Zheng, and C. Jiang, "Incremental modeling of a new high-order polynomial surrogate model," Applied Mathematical Modelling, vol. 40, no. 7-8, pp. 4681-4699, 2016.

[44] R. Ahlfeld, B. Belkouchi, and F. Montomoli, "SAMBA: sparse approximation of moment-based arbitrary polynomial chaos," Journal of Computational Physics, vol. 320, pp. 1-16, 2016.

[45] W. Zhu, Y. Hu, N. Chen, J. Liu, and M. Beer, “A fuzzy and random moment-based arbitrary polynomial chaos method for response analysis of composite structural-acoustic system with multi-scale uncertainties," Applied Acoustics, vol. 177, Article ID 107913, 2021.

[46] D. Xiu and J. S. Hesthaven, "High-order collocation methods for differential equations with random inputs," SIAM Journal on Scientific Computing, vol. 27, no. 3, pp. 1118-1139, 2005.

[47] F. Nobile, R. Tempone, and C. G. Webster, "A sparse grid stochastic collocation method for partial differential equations with random input data," SIAM Journal on Numerical Analysis, vol. 46, no. 5, pp. 2309-2345, 2008.

[48] D. Xiu, "Efficient collocational approach for parametric uncertainty analysis," Communications in Computational Physics, vol. 2, no. 2, pp. 293-309, 2007.

[49] J. Wu, Z. Luo, N. Zhang, and W. Gao, "A new sequential sampling method for constructing the high-order polynomial surrogate models," Engineering Computations, vol. 35, no. 2, pp. 529-564, 2018.

[50] J. Chen, B. Xia, and J. Liu, "A sparse polynomial surrogate model for phononic crystals with uncertain parameters," Computer Methods in Applied Mechanics and Engineering, vol. 339, no. 1, pp. 681-703, 2018.

[51] R. Tempone and S. Wolfers, "Smolyak's algorithm: a powerful black box for the acceleration of scientific computations,"
Lecture Notes in Computational Science and Engineering, Sparse Grids and Applications, Miami, FL, USA, pp. 201-228, 2018.

[52] A. L. A. Haji, H. Harbrecht, M. D. Peters, and M. Siebenmorgen, "Novel results for the anisotropic sparse grid quadrature," Journal of Complexity, vol. 47, pp. 62-85, 2018.

[53] J. He, S. Gao, and J. Gong, "A sparse grid stochastic collocation method for structural reliability analysis," Structural Safety, vol. 51, pp. 29-34, 2014.

[54] M. E. Johnson, L. M. Moore, and D. Ylvisaker, "Minimax and maximin distance designs," Journal of Statistical Planning and Inference, vol. 26, no. 2, pp. 131-148, 1990.

[55] M. D. Morris and T. J. Mitchell, "Exploratory designs for computational experiments," Journal of Statistical Planning and Inference, vol. 43, no. 3, pp. 381-402, 1992. 